\title{
Effect of an Advanced Pharmacy Practice Experience on Medication Therapy Management Services in a Centralized Retail Pharmacy Program
}

\author{
Joseph P. Vande Griend, PharmD; Melissa Rodgers, PharmD; and Wesley Nuffer, PharmD
}

\begin{abstract}
BACKGROUND: Medication therapy management (MTM) delivery is increasingly important in managed care. Successful delivery positively affects patient health and improves Centers for Medicare \& Medicaid Services star ratings, a measure of health plan quality. As MTM services continue to grow, there is an increased need for efficient and effective care models. The primary objectives of this project were to describe the delivery of MTM services by fourth-year Advanced Pharmacy Practice Experience (APPE) students in a centralized retail pharmacy system and to evaluate and quantify the clinical and financial contributions of the students. The secondary objective was to describe the engagement needed to complete comprehensive medication reviews (CMRs) and targeted interventions.
\end{abstract}

PROGRAM DESCRIPTION: From May 2015 to December 2015, thirty-five APPE students from the University of Colorado Skaggs School of Pharmacy provided MTM services at Albertsons Companies using the OutcomesMTM and Mirixa platforms. Students delivered patient care services by phone at the central office and provided face-to-face visits at pharmacies in the region. With implementation of the MTM APPE in 2015, the team consisted of 2 MTM pharmacists and pharmacy students, as compared with 1 MTM pharmacist in 2014. The number of CMRs and targeted interventions completed and the estimated additional revenue generated during the 2015 time period were compared with those completed from May through December 2014. The patient and provider engagement needed to complete the CMRs and targeted interventions was summarized.

OBSERVATIONS: 125 CMRs and 1,918 targeted interventions were billed in 2015, compared with 13 CMRs and 767 targeted interventions in 2014. An estimated $\$ 16,575-\$ 49,272$ of additional revenue was generated in 2015. To complete the interventions in 2015, the team engaged in 1,714 CMR opportunities and 4,686 targeted intervention opportunities.

IMPLICATIONS/RECOMMENDATIONS: In this MTM rotation, students provided real-life care to patients, resulting in financial and clinical contributions. This model of education and care delivery can be replicated in the community pharmacy or managed care setting. APPE students are an important component of this model of care delivery, particularly when considering the level of patient engagement needed to complete MTM interventions.

J Manag Care Spec Pharm. 2017;23(5):561-65

Copyright $\odot 2017$, Academy of Managed Care Pharmacy. All rights reserved.

\section{What is already known about this subject}

Medication therapy management (MTM) in the community pharmacy setting can improve patient health outcomes, increase health plan quality indicators, and decrease health care costs. At best, the reimbursement for MTM delivery in the community pharmacy appears to be sufficient to sustain the salary of a pharmacist but does not generate additional revenue and does not cover overhead.

Advanced Pharmacy Practice Experience (APPE) students have been shown to contribute to the delivery of MTM and clinical care.

\section{What this study adds}

The addition of APPE students to a centralized MTM model may increase the completion of CMRs and targeted interventions and generate additional revenue.

Many patients and providers need to be engaged in order to complete CMRs and targeted interventions.

Using APPE students in a centralized MTM model could be replicated to enhance MTM productivity and provide a learning environment for APPE students.

$\mathrm{M}$ edication therapy management (MTM) can improve patient health outcomes, increase health plan quality indicators, and decrease health care costs. ${ }^{1-3}$ Successful completion of MTM is increasingly important in the evolving health care quality environment. The Centers for Medicare \& Medicaid Services (CMS) now include the completion of comprehensive medication reviews (CMRs) for Medicare patients as a component of the CMS star ratings program. ${ }^{4}$ In addition, several other components of the star ratings program can be addressed by pharmacists with MTM interventions, including adherence, CMR completion rate, blood pressure and blood sugar control, and receipt of annual influenza vaccine. Failure of a health plan to complete CMRs or improve medication management metrics can reduce the health plan's star rating; health plans with a higher star rating have greater opportunity to enroll patients and may be eligible for bonus payments, as well as rebates to be used to enhance plan offerings for patients. ${ }^{5}$ It is well established in the literature that pharmacists are able to provide these services to patients and 
achieve positive outcomes. ${ }^{1,2,6-10}$ Third-party payers are applying increasing pressure to community pharmacies to provide MTM services that include CMRs and targeted interventions with the goal of improving patient care as well as the plan's star rating.

The demand to provide CMRs and targeted interventions is a positive opportunity for the pharmacy profession, but it also presents challenges. At best, the reimbursement for MTM delivery in the community pharmacy appears to be sufficient to sustain the salary of a pharmacist, but it does not generate additional revenue and would not cover overhead. ${ }^{11,12}$ For chain pharmacies, it can be difficult to provide consistent store-level MTM services across a broad geographic region. In the existing environment, effective and cost-efficient MTM care delivery models are needed. Using pharmacy students to deliver MTM services may increase the potential for additional revenue, while reducing the burden of increased labor. ${ }^{12}$ Pharmacy students, particularly fourth-year students in Advanced Pharmacy Practice Experience (APPE) programs, have been shown to contribute to the delivery of MTM and clinical care. ${ }^{13-18}$ As MTM in the community pharmacy setting continues to expand, there is an increasing need for MTM-trained, entry-level pharmacists. Incorporating APPE students might be a care delivery model that could increase the efficiency and effectiveness of a community pharmacy MTM program, while also training the students to effectively deliver MTM services after graduation.

The primary objectives of this project were to (a) describe an MTM service delivered telephonically by APPE students, using a centralized model in a retail pharmacy chain, and (b) evaluate and quantify the clinical and financial contributions of students to MTM delivery across the company's stores. In order to further define the importance and need for studentdelivered MTM, the secondary objective was to describe the level of engagement needed for MTM completion of CMRs and targeted interventions.

\section{Program Description}

The University of Colorado Skaggs School of Pharmacy and Pharmaceutical Sciences designed a focused, 3-week MTM APPE in collaboration with external community pharmacy partners. For this experience, all students completed the American Pharmacists Association (APhA) Delivering MTM Services coursework after completion of the third year of the pharmacy program, before the beginning of the APPE year. One of the external community pharmacy partners, Albertsons Companies, used a centralized model consisting of several APPE students and 2 MTM pharmacists to deliver and document MTM services using the OutcomesMTM and Mirixa platforms. These platforms link community pharmacies with contracted third-party plans to alert the individual pharmacist of opportunities to improve a patient's medication management and to be reimbursed for care delivery by the third party.
At the start of each 3-week MTM APPE, students were provided an orientation to MTM delivery and education regarding workflow. Each APPE student was provided a workstation at the central corporate location to deliver patient care services by phone. A specialized MTM pharmacist managed the students' daily activities and workload and provided oversight for care provided. The specialized MTM pharmacist used the OutcomesMTM and Mirixa platforms to identify available MTM opportunities in the Albertsons Companies region, including targeted interventions and CMRs, and provided a list of these opportunities to the APPE students. The majority of targeted interventions focused on improving medication adherence; other common targeted interventions included recommending medications that are indicated for the patient but not currently prescribed, reducing high-risk medication use in older adults, and switching from high-cost to lower-cost alternatives.

The students were responsible for contacting patients and completing the interventions. Students also contacted patients and set up appointments for CMRs at the patients' local pharmacies. For some of the CMR appointments, the APPE student traveled to the local pharmacy to provide the service with the patient. CMRs were provided face-to-face for OutcomesMTM, since this was required by this platform during that time. Targeted interventions typically took 10 minutes or less to complete, whereas CMRs typically took 45 minutes to 1 hour to complete. The CMRs could also require travel to the local stores throughout the Albertsons Companies region in the Colorado area. Delivery of the CMR at the local pharmacy was not associated with the dispensing of a medication but was intended to improve the face-to-face CMR completion rate by enhancing patient convenience and comfort. In general, students provided approximately $90 \%$ of MTM care by phone and $10 \%$ of MTM care face-to-face at the patient's pharmacy. Overall, students spent $50 \%$ of their time preparing for the outreach and attempting to contact the patient and $50 \%$ of their time delivering the MTM, documenting the care, and following up with the provider.

To evaluate and quantify the clinical and financial contributions of the students to MTM delivery, we compared the MTM interventions completed by the centralized team before the MTM APPE rotation was implemented (May 2014 through December 2014) with the contributions of the centralized team during the first 5 MTM APPE rotations (May 2015 through December 2015). In 2014, there was 1 pharmacist providing all MTM services for the centralized team. In 2015, there was 1 pharmacist and the APPE students completing CMRs and 2 pharmacists and the APPE students completing targeted interventions. The revenue generated from CMRs and targeted interventions was retrospectively estimated using national MTM reimbursement averages available internally for Albertsons Companies. During care delivery, the 


\begin{tabular}{|c|c|c|}
\hline $\begin{array}{ll}\text { TABLE } 1) & \mathrm{N} \\
\mathrm{fc} & \mathrm{f}\end{array}$ & \multicolumn{2}{|c|}{$\begin{array}{l}\text { Number of Patients Engaged and } \\
\text { Number of Interventions Completed } \\
\text { for OutcomesMTM and Mirixa Claims }\end{array}$} \\
\hline & 2014 & 2015 \\
\hline \multicolumn{3}{|l|}{ Targeted intervention } \\
\hline Number engaged & 767 & 3,319 \\
\hline Number completed (\%) & $411 \quad(53.6)$ & $1,918 \quad(57.8)$ \\
\hline \multicolumn{3}{|c|}{ Comprehensive medication review } \\
\hline Number engaged & 13 & 396 \\
\hline Number completed (\%) & $13(100)$ & $125 \quad(31.6)$ \\
\hline
\end{tabular}

number of patients the centralized team engaged with in order to complete CMRs and targeted interventions from May 2015 through December 2015 was documented by a specialized MTM pharmacist as part of the ongoing continuous quality improvement of the MTM service, including the reasons for engagement but not completion. At a minimum, engagement consisted of the students and/or pharmacists attempting to contact the patients and/or providers to complete the MTM care delivery. If they were successful at contacting the patients and/ or providers, they would communicate the recommendations to the provider and complete the intervention. The data regarding the number of patients engaged in order to complete MTM care delivery were only available from OutcomesMTM claims and were summarized and evaluated for this project. Descriptive statistics were used to summarize data and are presented as percentages. The Colorado Multiple Institutional Review Board designated this project as nonhuman subject research.

A total of 35 APPE students completed the MTM APPE rotation at Albertsons Companies from May 2015 through December 2015. This established a centralized team of 3-4 students per 3-week cycle and 2 pharmacists to provide MTM services in 2015. During the 2014 time period, when the centralized team consisted of only 1 pharmacist and no students, there were 13 CMRs completed and 411 targeted interventions completed (Table 1). All attempted CMRs were successfully completed. An additional 356 targeted interventions were attempted but were not completed with the patient and/or provider; these were billed at the lowest level. The pharmaciststudent team in 2015 completed 125 CMRs and 1,918 targeted interventions. An additional $271 \mathrm{CMRs}$ and 1,401 targeted interventions were attempted but were not completed and were billed at the lowest level. This equates to a $962 \%$ increase in CMR completion, and a $467 \%$ increase in targeted intervention completion, when comparing 2014 with 2015.

Using national MTM reimbursement averages, the centralized team in 2015 generated an estimated $\$ 16,575$ to $\$ 49,272$ of additional revenue compared with the 2014 centralized team. For OutcomesMTM, the number of interventions that focused on Pharmacy Quality Alliance measures were available in the 2015 time period (Table 2), as well as the reasons

\section{TABLE 2 Targeted PQA Measures for} OutcomesMTM Claims in 2015

\begin{tabular}{l|c|rc}
\hline PQA Measure & Engagement, $\mathbf{n}$ & \multicolumn{2}{|c}{ Completion, $\mathbf{n}(\%)$} \\
\hline Proportion of days covered & 1,713 & 1,138 & $(66.4)$ \\
\hline High-risk medications in the elderly & 368 & 32 & $(8.7)$ \\
\hline Statin use in diabetes & 72 & 9 & $(12.5)$ \\
\hline Drug-drug interactions & 12 & 0 & $(0.0)$ \\
\hline CMR completion rate & 1,563 & 72 & $(4.6)$ \\
\hline
\end{tabular}

$C M R=$ comprehensive medication review; $P Q A=$ Pharmacy Quality Alliance.

why the patient and/or provider engagement occurred, but the targeted intervention was not completed (Table 3). These data were not collected in 2014 so were not analyzed.

\section{Observations}

The pharmacist-student centralized team in 2015 was more productive in completing CMRs and targeted interventions compared with the 2014 team. For CMRs, the increase in completion can be attributed to the addition of APPE students, since there was the same pharmacist time allocated in 2014 as in 2015 . For targeted interventions, the increase in completion can be attributed to the increase in allocated pharmacist time and to the addition of APPE students to the centralized team in 2015.

The centralized team had to engage many patients in order to complete CMRs and targeted interventions. Looking at the 2015 data from OutcomesMTM and Mirixa presented in Table 1, the number needed to engage (NNE) in order to complete 1 CMR was 13.7, whereas the NNE to complete 1 targeted intervention was 2.4. This means that in 2015 the centralized team needed to work with 13.7 patients to contact, schedule, and complete 1 CMR and 2.4 patients and/or providers in order to complete 1 targeted intervention. It is interesting that, in 2014, all CMRs attempted were successfully completed. Interpreting the 2015 data from OutcomesMTM presented in Table 2, the MTM intervention of proportion of days covered was completed most frequently, with an NNE of 1.5 to complete 1 intervention. These targeted interventions only require contacting the patient in order to identify and resolve nonadherence and do not require contacting the provider. Table 3 shows that the primary reason this intervention was not completed was the inability to successfully reach the patient, followed by patient refusal.

Reducing the use of high-risk medications in the elderly, increasing statin use in diabetes, and completing CMRs proved to be more difficult to successfully complete. The NNE for these interventions was 11.5, 8.0, and 21.7, respectively. For highrisk medications, a primary reason for nonimplementation was because the patient/provider could not be reached. Excluding when the patient/provider could not be reached, $78.4 \%$ of the remaining interventions not completed were because of prescriber refusal. Prescriber refusal provides an opportunity to improve effectiveness of the intervention. Available data 
TABLE 3 Reasons for Engagement Without Completion for PQA Measures in 2015

\begin{tabular}{|c|c|c|c|c|c|c|c|}
\hline \multirow{3}{*}{$\begin{array}{l}\text { PQA Measure Not Completed } \\
\text { Proportion of days covered } \\
\text { High-risk medications in the elderly }\end{array}$} & \multirow{3}{*}{$\begin{array}{c}\begin{array}{c}\text { Total } \\
\mathbf{n}\end{array} \\
575 \\
336 \\
\end{array}$} & \multicolumn{2}{|c|}{$\begin{array}{c}\text { Patient Refusal } \\
\text { n (\%) }\end{array}$} & \multicolumn{2}{|c|}{$\begin{array}{c}\text { Could Not Reach } \\
\text { n (\%) }\end{array}$} & \multirow{2}{*}{\multicolumn{2}{|c|}{$\begin{array}{c}\begin{array}{c}\text { Prescriber Refusal } \\
\text { n (\%) }\end{array} \\
\text { N/A } \\
\end{array}$}} \\
\hline & & 179 & $(31.1)$ & 396 & $(68.9)$ & & \\
\hline & & 35 & $(10.0)$ & 174 & $(51.8)$ & 127 & $(37.8)$ \\
\hline Statin use in diabetes & 63 & 4 & $(6.3)$ & 17 & $(27.0)$ & 42 & $(66.7)$ \\
\hline Drug-drug interactions & 12 & 2 & $(16.7)$ & 3 & $(25.0)$ & 7 & $(58.3)$ \\
\hline
\end{tabular}

suggest that providers are reluctant to stop potentially harmful medications for several reasons, including a lack of awareness of potential harm from the medication, lack of clinical knowledge in the area together with poor communication between specialists and primary care providers, and limitations in working with patients to address the medication change. ${ }^{19}$ Pharmacists and students providing MTM may be able to explore these barriers in order to improve intervention effectiveness.

For statin use in diabetes, the majority of interventions that were not implemented were also because of refusal by the prescriber (66.7\%; Table 3). Similar prescriber barriers likely exist with implementation of this recommended intervention. In addition, providers are often aware of the need for statin use in diabetes, but patient limitations, such as previous statin intolerance, can limit implementation.

CMRs appear to be difficult to complete. For OutcomesMTM claims, it was required to complete CMRs face-to-face with the pharmacist in 2015. With Mirixa, CMRs could be completed over the phone. When combining data from OutcomesMTM and Mirixa, the overall NNE for CMRs was 13.7. For OutcomesMTM only, the NNE was 21.7. This suggests difficulty with completing CMRs face-to-face, likely because of difficulty in convincing patients to come into the pharmacy to meet and discuss their medications. This situation is particularly difficult with a centralized model, where the MTM pharmacist is providing care offsite from the local pharmacy and does not have a relationship with the patient.

\section{Strengths and Limitations}

The strength of this project is that it describes real-world implementation of a successful model that incorporated APPE students to increase the productivity of an MTM program. The MTM environment was similar when comparing 2014 data with 2015 data, with the only change being the addition of 1 MTM pharmacist and the APPE students.

This project has the inherent limitations of a pre-post study design. With the data available for targeted intervention completion in 2015, it was not possible to separate the contributions of the additional 1 full-time pharmacist from those of the students. Nevertheless, the substantial increase in CMRs and targeted interventions demonstrates the value that APPE students can bring to a pharmacy team.

\section{Implications/Recommendations}

An MTM care delivery model using APPE students as part of a centralized team was successfully established at Albertsons Companies. The students provided MTM care, which was observed to increase targeted intervention and CMR completion, and generated additional revenue. This type of care delivery model has the benefit of increasing the skills of pharmacy students, training them in MTM delivery, and generating revenue. The students were not paid for the work they provided and did require additional training, oversight, and management. If the students were not available to provide this MTM care, it is possible that additional pharmacist resources would have been needed to maintain the level of productivity seen in 2015 .

On an individual level, students often do not function as licensed pharmacy practitioners and may require significant oversight to deliver high-level care. However, this project demonstrated that, as a whole, the delivery of care by APPE students at Albertsons Companies was a valuable function. Replicating this care delivery model in other pharmacy settings may represent a business case opportunity to enhance MTM productivity and provide a learning environment for APPE students.

Significant patient and provider engagement was needed for MTM completion. Strategies and models are needed to increase completion rate of MTM services, particularly for CMRs. The national average for CMR completion rate was approximately 21\% for Medicare Advantage Prescription Drug plans in 2014.20 Our project had a CMR completion rate of only $4.6 \%$ for the data available with OutcomesMTM claims, which similarly demonstrates difficulty with completing CMRs. Since APPE students provided MTM care delivery in this project, there may be a need to provide additional education and emphasis regarding patient and provider engagement in the curriculum, with the goal of enhancing patient engagement that leads to increased implementation of medication interventions. Given the data showing low CMR completion rates, strategies to further increase efficiency and effectiveness of MTM care delivery are needed. 


\section{Authors}

JOSEPH P. VANDE GRIEND, PharmD, Department of Clinical Pharmacy, University of Colorado Skaggs School of Pharmacy and Pharmaceutical Sciences, and Department of Family Medicine, University of Colorado School of Medicine, Aurora. MELISSA RODGERS, PharmD, and WESLEY NUFFER, PharmD, Department of Clinical Pharmacy, University of Colorado Skaggs School of Pharmacy and Pharmaceutical Sciences, Aurora.

AUTHOR CORRESPONDENCE: Joseph P. Vande Griend, PharmD, Associate Professor, Departments of Clinical Pharmacy and Family Medicine, University of Colorado School of Medicine, Mail Stop C238, 12850 E. Montview Blvd., Rm. V20-2127, Aurora, CO 80045. E-mail: joseph.vandegriend@ucdenver.edu.

\section{DISCLOSURES}

No outside funding supported this research. The authors have no conflicts of interest to disclose related to this work.

All authors contributed to study concept and design. Rodgers collected the data, and data interpretation was performed by Vande Griend, along with Rodgers and Nuffer. The manuscript was written and revised primarily by Vande Griend, along with Nuffer and Rodgers.

This project was presented at the Pharmacy Quality Alliance Annual Meeting in Arlington, Virginia, in May 2016.

\section{REFERENCES}

1. Fera T, Bluml BM, Ellis WM. Diabetes Ten City Challenge: final economic and clinical results. J Am Pharm Assoc (2003). 2009;49(3):383-91.

2. Cranor CW, Bunting BA, Christensen DB. The Asheville Project: longterm clinical and economic outcomes of a community pharmacy diabetes care program. J Am Pharm Assoc (Wash). 2003;43(2):173-84.

3. Branham AR, Katz AJ, Moose JS, Ferreri SP, Farley JF, Marciniak MW. Retrospective analysis of estimated cost avoidance following pharmacist-provided medication therapy management services. J Pharm Pract. 2013;26(4)420-27.

4. Centers for Medicare \& Medicaid Services. Medicare 2016 Part C and D star rating technical notes. First plan preview. Draft. August 5, 2015. Available at: https://www.cms.gov/Medicare/Prescription-Drug-coverage/ PrescriptionDrugCovGenIn/Downloads/2016-Technical-Notes-Preview1-v2015_08_05.pdf. Accessed March 25, 2017.

5. Centers for Medicare \& Medicaid Services. Advance notice of methodological changes for calendar year (CY) 2016 for Medicare Advantage (MA) capitation rates, Part C and Part D payment policies and 2016 call letter [Memo]. February 20, 2015. Available at: www.cms.gov/Medicare/HealthPlans/MedicareAdvtgSpecRateStats/Downloads/Advance2016.pdf. Accessed March 25, 2017.
6. Ramalho de Oliveira D, Brummel AR, Miller DB. Medication therapy management: 10 years of experience in a large integrated health care system. J Manag Care Pharm. 2010;16(3):185-95. Available at: http://www.jmcp.org/ doi/10.18553/jmcp.2010.16.3.185.

7. Fox D, Ried LD, Klein GE, Myers W, Foli K. A medication therapy management program's impact on low-density lipoprotein cholesterol goal attainment in Medicare Part D patients with diabetes. J Am Pharm Assoc (2003). 2009;49(2):192-99.

8. Barnett MJ, Frank J, Wehring H, et al. Analysis of pharmacist-provided medication therapy management (MTM) services in community pharmacies over 7 years. J Manag Care Pharm. 2009;15(1):18-31. Available at: http:// www.jmcp.org/doi/10.18553/jmcp.2009.15.1.18.

9. Peaslee A, Wickizer M, Olson J, Topp R. Impact of a combined value-based insurance design and medication therapy management program on diabetes medication adherence. J Manag Care Spec Pharm. 2016;22(11):1303-09. Available at: http://www.jmcp.org/doi/full/10.18553/ jmcp.2016.22.11.1303.

10. Brummel A, Lustig A, Westrich K, et al. Best practices: improving patient outcomes and costs in an ACO through comprehensive medication therapy management. J Manag Care Spec Pharm. 2014;20(12):1152-58. Available at: http://www.jmcp.org/doi/pdf/10.18553/jmcp.2014.20.12.1152.

11. Rhodes SA, Reynolds AE, Marciniak MW, Ferreri SP. Evaluating the economic impact of a targeted medication intervention program. J Pharm Pract. 2013;26(6):562-73.

12. McDonough RP, Harthan AA, McLeese KE, Doucette WR. Retrospective financial analysis of medication therapy management services from the pharmacy's perspective. J Am Pharm Assoc (2003). 2010;50(1):62-66.

13. Melody KT, Shah CJ, Patel J, Willey VJ. Implementation of a student pharmacist-run targeted medication intervention program. J Pharm Pract. 2017;30(1):109-14.

14. Hardin HC, Hall AM, Roane TE, Mistry R. An advanced pharmacy practice experience in a student-staffed medication therapy management call center. Am J Pharm Educ. 2012;76(6):110.

15. Hata M, Klotz R, Sylvies R, et al. Medication therapy management services provided by student pharmacists. Am J Pharm Educ. 2012;76(3):51.

16. McCollum M, Nuffer W, Ellis SL, Turner CJ. Physician acceptance of pharmacotherapy recommendations made by pharmacy students in a rural pharmacy-based diabetes care and education clinic. Am J Pharm Educ. 2009;73(2):24.

17. Shepler BM. Cost savings associated with pharmacy student interventions during APPEs. Am J Pharm Educ. 2014;78(4):71.

18. Woolley AB, Berds CAt, Edwards RA, Copeland D, DiVall MV. Potential cost avoidance of pharmacy students' patient care activities during advanced pharmacy practice experiences. Am J Pharm Educ. 2013;77(8):164.

19. Anderson K, Stowasser D, Freeman C, Scott I. Prescriber barriers and enablers to minimising potentially inappropriate medications in adults: a systematic review and thematic synthesis. BMJ Open. 2014;4(12):e006544.

20. Miller DE, Roane TE, Salo JA, Hardin HC. Evaluation of comprehensive medication review completion rates using 3 patient outreach models. J Manag Care Spec Pharm. 2016;22(7):796-800. Available at: www.jmcp.org/ doi/10.18553/jmcp.2016.22.7.796. 